\title{
ARAÑAS (ARACHNIDA: ARANEAE) PELIGROSAS DE LA REGIÓN DE MAGALLANES'1.
}

\author{
DANGEROUS SPIDERS (ARACHNIDA: ARANEAE) FROM THE MAGELLAN REGION.
}

Se consideran animales potencialmente peligrosos todos los que perteneciendo a la fauna salvaje, siendo utilizados como animales domésticos o de compañía, con independencia de su agresividad, pertenecen a especies o razas que tengan capacidad de causar la muerte o lesiones a las personas o a otros animales y daños a las cosas (Melic 1995).

Todas las arañas, excepto la pequeña familia Uloboridae (unas 200 especies), son venenosas, es decir producen sustancias tóxicas (com. pers. A. Melic). Sin embargo, solamente una pequeña parte de éstas son capaces de producir daño a los humanos, pese a esto las arañas se encuentran dentro de los animales más temidos por los humanos desarrollando en algunas personas casos extremos de aracnofobia.

Chile cuenta constantemente con una gran cantidad de casos de mordeduras de arañas, siendo normal la aparición de noticias al respecto en los distintos medios de comunicación nacionales.

En Chile la arañas más conocidas por su peligrosidad son las del género Loxosceles Heineken \& Lowe, 1832 (Sicariidae), principalmente Loxosceles laeta (Nicolet, 1849), estas arañas son llamadas, comúnmente "arañas de los rincones" seguida por las especies del género Latrodectus Walckenaer,
1805 (Theridiidae) conocidas comúnmente como "viudas negras", "arañas del trigo" o "arañas de poto colorado", en el caso de ambos géneros se han registrado mordeduras con efectos fatales generando en la comunidad la impresión de que éstas son las únicas arañas peligrosas de Chile. Canals et. al. (2004) proponen para Chile como arañas peligrosas a las pertenecientes a los géneros Loxosceles, Latrodectus y Lycosa Latreille, 1804; sin embargo, existen otros géneros de arañas presentes en el país que son consideradas peligrosas para los humanos, como es el caso del género Steatoda Sundevall, 1833 (Theridiidae) conocidas como "falsas viudas" y el género Sicarius Walckenaer, 1847 (Sicariidae) cuyo nombre vulgar es "arañas sicario".

La mayoría de los registros de mordeduras de arácnidos en Chile se refieren a Loxosceles, seguidos por Latrodectus (Schenone 2003) y Lycosa (Donoso 1948). Este último autor responsabiliza de lesiones necróticas cutáneas a L. murina Nicolet, 1849, actualmente L. implacida Nicolet, 1849.

La principal razón de que estos géneros de arañas sean los únicos considerados peligrosos puede deberse a tres razones: son los únicos en que los ejemplares mordedores han sido taxonómicamente bien identificados, a que los pacientes normalmente

1 Corresponde al proyecto "Arañas peligrosas de Magallanes" del programa "Transferencia financiamiento tesis universitaria de interés regional. XII región" del Gobierno Regional de Magallanes y la Antártica Chilena.

2 Grupo Entomon, Laboratorio de Entomología, Instituto de la Patagonia, Universidad de Magallanes. Casilla 113-D Punta Arenas, Chile.ed.faundez@gmail.com 
no llevan al servicio de salud las arañas mordedoras o a que el personal médico no las haya sabido identificar (Schenone 2003), y por tanto cabe dudar con respecto a cuáles son las arañas efectivamente responsables de las lesiones.

Los venenos de las arañas peligrosas chilenas pueden clasificarse en dos tipos, los necróticos producidos por Loxosceles y Lycosa (esta última con mucho menor peligrosidad que Loxosceles) e incluiremos dentro de éste grupo a Sicarius, dado que por su parentesco con Loxosceles es muy probable que su mordedura tenga los mismos efectos y posiblemente mayores; a pesar de no existir registros en nuestro país es muy posible que muchos de aquellos sin la identificación del arácnido responsable de la mordedura, y asignados a Loxosceles puedan pertenecer a éste género. El otro grupo corresponde al de los venenos neurotóxicos en donde encontramos al género Latrodectus y también al género Steatoda, aunque para Chile no existen casos formalmente registrados por mordedura de Steatoda pero al igual que el caso anterior posiblemente muchas de las mordeduras sin confirmación del arácnido asignadas a Latrodectus pueden corresponder a Steatoda, idea que se potencia en este caso debido a que especies como Steatoda grossa (C. L. Koch, 1838) son comunes habitantes en las casas aumentando las probabilidades de accidentes; también para esta especie existen en Chile casos de mordeduras registrados que no han sido publicados produciendo la misma sintomatología que Latrodectus (com. pers. C. Xavier Pérez).

De este modo concluimos que los géneros de arañas peligrosas de Chile son cinco los cuales presentamos en la siguiente lista (para efectos taxonómicos se sigue a Platnick (2009)):

\section{GÉNEROS DE ARAÑAS}

PELIGROSAS DE CHILE

Familia Lycosidae Sundevall, 1833

Género Lycosa Latreille, 1804

Familia Sicariidae Keyserling, 1880

Género Loxosceles Heineken \& Lowe, 1832
Familia Theridiidae Sundevall, 1833

\section{Género Latrodectus Walckenaer, 1805}

\section{Género Steatoda Sundevall, 1833}

El conocimiento de la aracnofauna de Magallanes es escaso y se debe principalmente a los resultados de expediciones antiguas en Chile austral, y de éstas para Magallanes se desprenden los trabajos de Simon (1884, 1886, 1887, 1895, 1896, 1902, 1903) y Tullgreen (1901); posteriormente Cekalovic (1976) publica su catálogo de los arácnidos de Magallanes contabilizando 121 especies de arañas para la región, sin que existan más trabajos posteriores referentes exclusivamente a arácnidos de Magallanes hasta que Faúndez (2007) trata al género Steatoda.

Prácticamente no existe información en Magallanes sobre arañas peligrosas y la percepción general es de que no están presentes en la región, esto se debe probablemente a la ausencia de Loxosceles que es el género de araña peligrosa más común del país, para el cual sólo hemos registrado algunos ejemplares aislados pertenecientes a L. laeta (Nicolet) que han venido en equipajes y encomiendas, sin que hayan logrado naturalizarse en la zona; pero aunque Loxosceles se encuentra ausente en la región los otros cuatro géneros de arañas peligrosas si lo están, cuyo elenco sistemático damos a conocer a continuación (para efectos taxonómicos se sigue a Platnick (2009)):

\section{ELENCO SISTEMÁTICO DE ARAÑAS PELIGROSAS DE MAGALLANES}

Familia Lycosidae Sundevall, 1833

Género Lycosa Latreille, 1804 (Presente en las siguientes provincias: Antártica Chilena (comuna de Cabo de Hornos) [referentes: Cekalovic 1976, Casanueva 1980]; Magallanes [referentes: Cekalovic 1976, Casanueva 1980, colección del autor]; Tierra del Fuego [referentes: Cekalovic 1976, Casanueva 1980, colección del autor]; Última Esperanza [referentes: Cekalovic 1976, Casanueva 1980, colección del autor]) 
Lycosa australis Simon, 1884

Lycosa implacida Nicolet, 1849

=L. murina Nicolet, 1849

Lycosa magallanica Karsch, 1880

= Lycosa ohlini Tullgreen, 1901

Lycosa patagonica Simon 1886

Lycosa serranoa Tullgreen 1901

Familia Sicariidae Keyserling, 1880

Género Sicarius Walckenaer, 1847 (Presente en la provincia de Magallanes [referente: Cekalovic 1976])

Sicarius terrosus (Nicolet, 1849)

Familia Theridiidae Sundevall, 1833

Género Latrodectus Walckenaer, 1805 (Presente en las siguientes provincias: Última Esperanza [referente: Cekalovic 1976]; Tierra del Fuego [referente: colección del autor])

\section{Latrodectus curacaviensis (Muller, 1776)}

Género Steatoda Sundevall, 1833 (Presente en las siguientes provincias: Magallanes [referente: Faúndez 2007]; Última Esperanza [referente: Faúndez 2007])

Steatoda ancorata (Holmberg, 1876)

= Asagena patagonica Tullgren, 1901

Steatoda grossa (C. L. Koch, 1838)

Steatoda sabulosa (Tullgren, 1901)

De este modo concluimos que en la región de Magallanes habitan 10 especies de arañas peligrosas, distribuidas en cuatro géneros y tres familias, donde destaca la familia Lycosidae como la más numerosa representada por el género Lycosa y se encuentra presente en todas las provincias de la región; de las 10 especies la que presenta un mayor riesgo de salud pública es $S$. grossa (C. L. Koch) debido a que es un común habitante en las casas y la posibilidad de un accidente es mayor (cabe destacar que hemos encontrado ejemplares de Lycosa en casas pero su ocurrencia es menor), a pesar de ser una especie recientemente arribada a la región (Faúndez 2007) colectas posteriores han permitido confirmar su naturalización en la zona, hecho de importancia ya que deja latente la posibilidad de nuevos arribos de arácnidos peligrosos por lo que se hace necesario un permanente monitoreo.

Finalmente con el objeto de mejorar el conocimiento sobre éstas arañas y proveer una herramienta que permita precisar mejor cuales son los arácnidos responsables de mordeduras en el país se entrega una clave pictórica para los géneros de arañas peligrosas de Chile.

\section{AGRADECIMIENTOS}

Deseamos manifestar nuestro agradecimiento a Vicente Pérez D'A. por leer críticamente el texto, a Antonio Melic por atender nuestras consultas, a C. Xavier Pérez por su colaboración con figuras para la clave pictórica y sus valiosos comentarios, a Gianfranco Bevilacqua, Carlos Rojas y Julio Cabezas G. por sus aportes fotográficos para la clave pictórica.

\section{LITERATURA CITADA}

Canals, M. M. E. Casanueva, M. Aguilera. 2004. ¿Cuáles son las arañas peligrosas de Chile?. Rev. Méd. Chile. 132: 773-776.

Casanueva, M. E. 1980. Los licósidos de Chile. Estudio biológico y taxonómico por los métodos de sistemática alfa y taxonomía numérica (Araneae: Lycosidae). Gayana 42: 5-76.

Cekalovic, K. 1976. Catálogo de los Arachnida: Scorpiones, Pseudoscorpiones, Opiliones, Acari, Araneae y Solifugae de la XII Región de Chile, Magallanes incluyendo la Antártica chilena (Chile). Gayana. 37: 1-108.

Donoso, R. 1948. Consideraciones sobre aracnoidismo cutáneo en Chile. Apartado de los archivos uruguayos de medicina, cirugía, y especialidades. Tomo XXXIII 5-6: 184-206.

Faúndez E. I. 2007. Datos sobre las especies del género Steatoda Sundevall, 1833 (Arachnida: Theridiidae) de la región de Magallanes (Chile). Anales Instituto Patagonia. 35(1): 79-80. 
Melic, A. 1995. Animales venenosos: 1. Generalidades. 2. Invertebrados Terrestres. Boletin Sociedad Entomológica Aragonesa. 11: 23-31.

Platnick, N. I. 2009. The world spider catalog, version 9.5. American Museum of Natural History, online at http://research.amnh.org/entomology/spiders/catalog/index.html

Schenone, H. 2003. Cuadros tóxicos producidos por mordeduras de araña en Chile: latrodectismo y loxocelismo. Rev. Méd. Chile. 131: 473-444.

Simon, E. 1884. Arachnides recuellis par la Mission du Cap Horn 1882-1883. Bull. Soc. Zool. France. 9: 117-144.

Simon, E. 1886. Arachnides recuellis en 1882-1883 dans la Patagonie meridionale, de Santa Cruz a Punta Arenas, par M. E. Lebrun, attache comme naturaliste a la Mission de passage de Venus. Bull. Soc. Zool. France. 11: 558-577.
Simon, E. 1887. Arachnides in Mission Scietifique du Cap Horn. Zool. 6: E1-E42.

Simon, E. 1895. Arachnides recuellis a la Terre de Feu par M. Carlos Backhausen. Anal. Mus. Nac. Buenos Aires (primera memoria) 4.

Simon, E. 1896. Arachnides recuellis a la Terre de Feu par M. Carlos Backhausen. Anal. Mus. Nac. Buenos Aires (segunda memoria) 5:167-172.

Simon, E. 1902. Arachniden, Acarien and Gonyleptiden in Ergebnisse der Hamburger Magalhenische Sammelreise 6 (4): 1-47.

Simon, E. 1903. Arachnides recueillis a la Tierre de Feu par Lehmann Nische. Etudes arachnologiques. Ann. Soc. Ent. France. 72: 310-313.

Tullgreen, A. 1901. Contribution to the knowledge of Magellan Territories. Suenka Expeditonen till Magellanslanderna. 2(10): 181-263. 


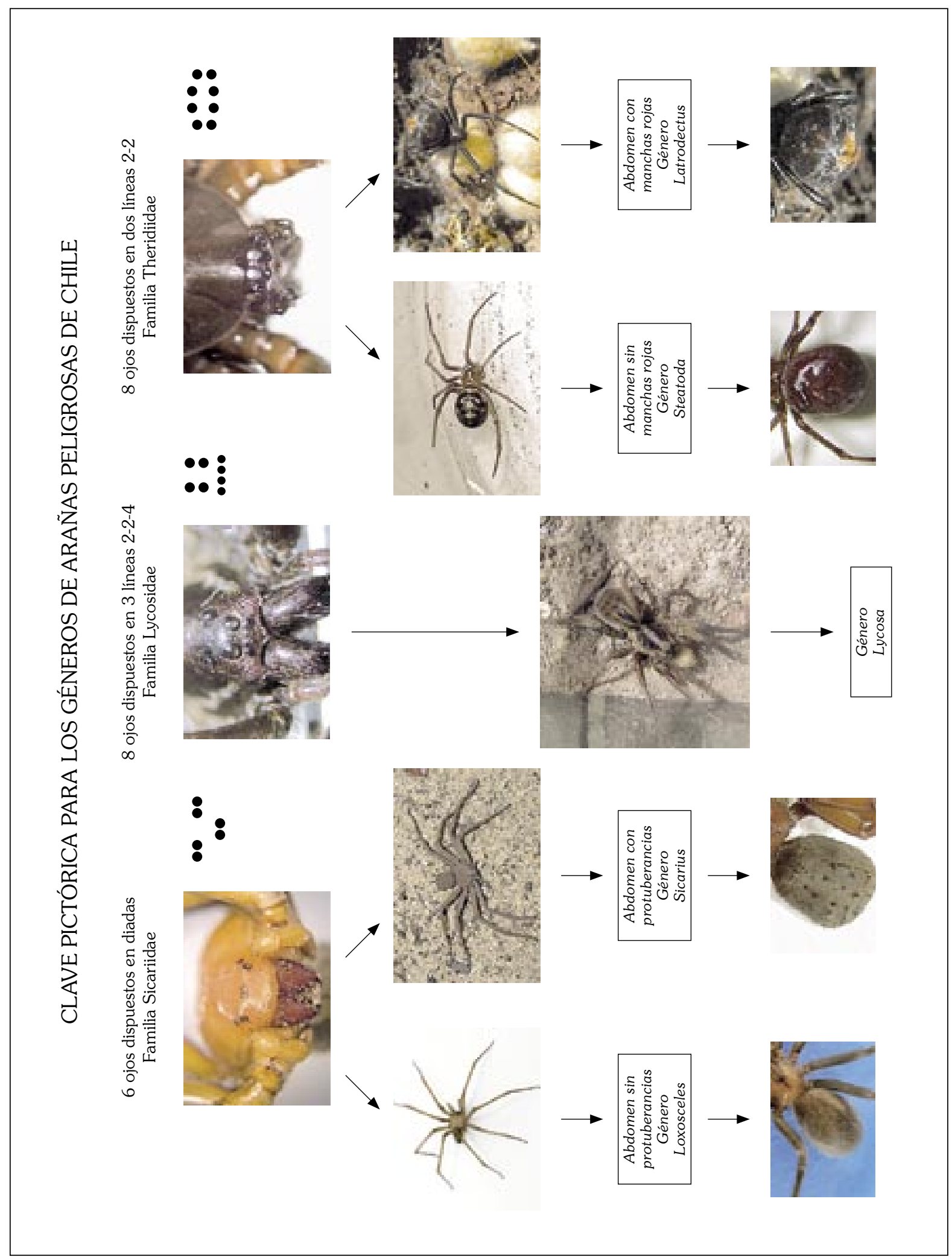


